\title{
Improved tissue integrity after alginate treatment in rat spinal cord injury: evidence from ex vivo diffusion tensor imaging
}

\author{
Ladislav Baciak ${ }^{1}$, Lubomir Melichercik ${ }^{1,2}$, Veronika Mate ${ }^{3}$, Adriana-Natalia Murgoci ${ }^{3,7}$, \\ Tomas Smolek ${ }^{3}$, Ivo Juranek ${ }^{4}$, Daniel Gogola ${ }^{6}$, Nikola Hudakova ${ }^{5}$, Milan Cizek ${ }^{5}$ and Dasa \\ Cizkova ${ }^{3,5}$ \\ ${ }^{1}$ Faculty of Chemical and Food Technology, Slovak University of Technology, Bratislava, Slovakia \\ ${ }^{2}$ Department of Radiology, University Hospital Bratislava, Bratislava, Slovakia \\ ${ }^{3}$ Institute of Neuroimmunology, Slovak Academy of Sciences, Bratislava, Slovakia \\ ${ }^{4}$ Centre of Experimental Medicine, Slovak Academy of Sciences, Bratislava, Slovakia \\ ${ }^{5}$ Centre of Experimental and Clinical Regenerative Medicine, University of Veterinary Medicine and Pharmacy in Kosice, \\ Kosice, Slovakia \\ ${ }^{6}$ Institute of Measurement Science, Slovak Academy of Sciences, Bratislava, Slovakia \\ ${ }^{7}$ Institute of Environmental Medicine, Toxicology Unit, Karolinska Institutet, Stockholm, Sweden
}

\begin{abstract}
Diffusion tensor imaging (DTI) is a magnetic resonance imaging technique used to characterize fibrous structures such as white matter in the central nervous system, including normal and spinal cord injury (SCI) conditions. Our aim was to evaluate the effect of alginate treatment in the rat SCI by DTI parametric measures. Ex vivo DTI data were collected by spin echo sequence with following parameters TR/TE: $2500 \mathrm{~ms} / 32 \mathrm{~ms}$ and b-value of $1500 \mathrm{~s} / \mathrm{mm}^{2}$. Main significant changes were found in fractional anisotropy (FA), and radial diffusivity (RD), between the saline- and alginatetreated group at the level of individual sections and whole spinal cord. Results indicate that ex vivo DTI can be used as a tool for tissue structure characterisation and both FA and RD as promising prognostic parameters of SCI treatment.
\end{abstract}

Key words: Diffusion tensor imaging — Spinal cord injury — Rat - Alginate treatment

Abbreviations: AD, axial diffusivity; DTI, diffusion tensor imaging; FA, fractional anisotropy; MD, mean diffusivity; RD, radial diffusivity; ROI, region of interest; SCI, spinal cord injury.

\section{Introduction}

Spinal cord injury (SCI), mostly caused by trauma, is a serious neurodegenerative condition accompanied by inflammation, demyelination, loss of neurons and glial cells. Patients suffering from SCI show limited functional recovery, which

Correspondence to: Dasa Cizkova, Centre of Experimental and Clinical Regenerative Medicine, University of Veterinary Medicine and Pharmacy in Kosice, Kosice, Slovakia

E-mail: cizkova.dasa@gmail.com often lead to partial or complete paralysis with prominent spasticity (Cizkova et al. 2020). Although much knowledge has been gained about the progression of SCI pathology affecting a large number of biochemical cascades, there is still no cure for spinal cord injury. One of the strategies for repairing chronic SCI is implantation of biomaterials that allow controlled, sustained and localized delivery of growth factors, leading to endogenous neural progenitor stimulation and enhanced sparing of spinal cord tissue (Cizkova et al. 2015). Great advances have been achieved in early diagnosis of SCI by using magnetic resonance imaging (MRI), which

(c) The Authors 2021. This is an open access article under the terms of the Creative Commons Attribution-NonCommercial 4.0 International License (https://creativecommons.org/licenses/by-nc/4.0/), which permits non-commercial use, distribution, and reproduction in any medium, provided the original work is properly cited. 
is the main radiographic method allowing visualization of injury characteristics such as bleeding and compression, but unable of evaluating the integrity of white matter tracts.

Diffusion tensor imaging (DTI) is a promising newer magnetic resonance (MR) modality that is sensitive to the diffusion of the water molecules. DTI provides supreme contrast over conventional MR techniques and is mostly used in studies of central nervous system tissue microstructure such as SCI (Kaushal et al. 2019). In spinal cord tissue the water diffusion is preferred in rostro-caudal direction, due to barriers applied in the transverse plane by myelin sheets. These spatial differences in water diffusion can be measured by the DTI. Following parameters can be derived from DTI data: fractional anisotropy (FA), which describes the degree of spatial inequality of water diffusion; axial diffusivity (AD) and radial diffusivity (RD) each of them describes water displacement along and across axon fibers, respectively. The last parameter is mean diffusivity (MD) which describes the average water displacement and is more or less the average of the previous two. In the grey matter the water diffusion is isotropic having $\mathrm{AD}$ and $\mathrm{RD}$ almost equal. On the contrary, in the white matter $\mathrm{AD}$ is much greater than RD. The diffusion is anisotropic due to myelin sheets architecture giving the characteristic contrast between those two tissues on DTI. For more details on the underlying mechanism please refer to review articles (Ellingson et al. 2008b; Smith et al. 2012).

Moreover, DTI derived parameters have been connected to specific white matter tissue damage. $\mathrm{AD}$ and $\mathrm{RD}$ have been shown to be related directly to axonal damage and demyelination, respectively. Even though this direct connection was challenged by later studies (Farrell et al. 2010; Talbott et al. 2016). On the contrary, FA is used as a global marker of spine tissue condition since it is insensitive to specific damage in the white matter. Nevertheless, these directional characteristics connected to tissue structure raised the potential of the DTI to be used in studies evaluating the underlying mechanisms of tissue damage and subsequent treatment efficacy assessment. And indeed DTI is the method of choice in vast in vivo and ex vivo studies of tissue characterisation after spinal cord injury. These studies are well summarized in various review articles (Smith et al. 2012; Kaushal et al. 2019). However, far less DTI studies were related to assessing the spinal cord tissue structure after the therapeutic intervention. Only recently diffusion tensor imaging was used to assess stem cell transplantation in the areas distal to the lesion in rat spinal cord injury model (Jirjis et al. 2017) and in the most recent study the DTI derived FA showed correlation with functional outcome after olfactory ensheathing cells administration in rats with contusive SCI (Gao et al. 2020).

It has to be noted that clinical use of DTI in SCI is still challenging and is limited by heterogeneity of local tissue (cerebrospinal fluid, vessels and bone) and consequent susceptibility and physiological motion artefacts. Further, the small cross-sectional dimension in preferred axial image orientation limits the spine cord investigation over the larger spine segment. Therefore, further improvement in imaging protocols is needed to address these issues together with ever increasing demand to higher signal to noise ratio and spatial resolution.

Our aim in the current study was to use DTI for evaluation of alginate treatment in rats after severe contusion injury. We have used a reproducible model of the clinically relevant balloon-induced dorsal lesion during chronic survival period (Vanický et al. 2001; Murgoci et al. 2019). We hypothesized that DTI-derived measures (namely FA, and RD) could serve as potential therapeutic markers that would reflect on applied therapy. In addition, we analysed DTI measures in two regions, dorsal column (DC) and entire spine segment to check for differences in DTI metrics with regard to injury location.

\section{Materials and Methods}

\section{Animals}

Male Wistar rats (200-250 g) were used for this experiment. Rats were divided into sham operated, SCI alginate-treated and SCI saline-treated groups $(n=6, n=12, n=11$, respectively). Rats were then allowed to survive for 14 weeks, after which ex vivo DTI scans were performed on the spinal cord. All procedures were approved by the Institutional Animal Care and Use Committee of the Slovak Academy of Sciences and with the European Communities Council Directive (2010/63/EU) regarding the use of animals in research and Slovak Law for Animal Protection 377/2012 and 436/2012. All experiments were approved by the State Veterinary and Food Committee of Slovak Republic (Ro-4081/17-221), and by the Ethics Committee of the Institute of Neuroimmunology, Slovak Academy of Sciences.

\section{SCI procedure}

The spinal cord damage was initiated by balloon-compression according to standardized model (Vanický et al. 2001; Murgoci et al. 2019). Rats were anesthetized with $1.5 \%$ to $2 \%$ isoflurane (Florane) while maintaining the temperature at $37^{\circ} \mathrm{C}$. The area of thoracolumbar spinal cord was shaved, sterilized with a povidone-iodine scrub pack. The rat was properly secured to a surgical board. After laminectomy, 4-French Fogarty Arterial Embolectomy Catheter was inserted into the epidural space at Th8-Th9 level of the vertebral column. After a stabilization period ( $2 \mathrm{~min}$ ) balloon was filled with $17 \mu \mathrm{l}$ of saline solution for $5 \mathrm{~min}$. Afterwards the saline was 
withdrawn and the catheter was removed from the vertebral canal. Finally, the tissue was closed by sutures. Compression induced severe SCI accompanied by initial complete paraplegia that was followed by a gradual mild recovery of locomotion. During the first week, manual bladder expression was performed twice a day until the bladder control was regained. Our consideration to use severe injury was to minimize the effect of spontaneous recovery often present in moderate contusion injuries (Vanický et al. 2001; Murgoci et al. 2019) that could confound the subsequent therapeutic effect.

\section{Alginate treatment}

Preparation of alginate biomaterial with the affinity-bound growth factors were performed according to our previous study (Grulova et al. 2015). The procedure involved preparing bioconjugates of trophic factors with alginate-sulfate and then mixing both bioconjugate solutions with the solution of a partially calcium-cross-linked alginate.

Seven days following SCI, rats were anesthetized (1.5-2\% isoflurane) and partial laminectomy was performed at Th612 level. Using a $50-\mu \mathrm{l}$ Hamilton syringe (30G needle, Cole Parmer, Anjou, Quebec) connected to an UltraMicroPump III with Micro4 Controller, 4-Channel (World Precious Instruments, Inc., Sarasota FL) and stereotactic device, 4 intraspinal injections/per animal were applied at the lesion site, identified by haemorrhage and slight atrophy. The intraspinal delivery was performed according to the procedure published in our previous study (Grulova et al. 2015). In the SCI group we have performed bilateral delivery of saline, or alginate that was positioned $1 \mathrm{~mm}$ from the spinal cord midline and injected at the depth of $1.8-2 \mathrm{~mm}$. The range between injections was $1 \mathrm{~mm}$, avoiding vessels. After injecting the dose of alginate, the needle was maintained in the tissue for an additional $30 \mathrm{~s}$. Animals were kept under postoperative care procedures until normal functions returned.

\section{Sample preparation}

The rats were deeply anesthetized by intraperitoneal injection of Zoletil (30 mg/kg, Virbac) and Xylariem $(10 \mathrm{mg} / \mathrm{kg}$, Ecuphar N.V) and intracardially perfused with $500 \mathrm{ml}$ of $4 \%$ paraformaldehyde (PFA) in $0.1 \mathrm{M} \mathrm{PBS}, 14$ weeks after SCI. Removed spinal cords were postfixed in $4 \%$ PFA at $4^{\circ} \mathrm{C}$ overnight, embedded in gelatin-egg albumin protein matrix $(10 \%$ ovalbumin, $0.75 \%$ gelatine) polymerized by glutaraldehyde (albumin from chicken egg white, grade II, Sigma-Aldrich) followed by postfixation in $4 \%$ PFA.

\section{Magnetic resonance imaging}

All MR measurements were performed on a $4.7 \mathrm{~T}$ directdrive horizontal scanner (Agilent, Yarton, UK) equipped with $12-\mathrm{cm}$ inner diameter gradient system (max gradient strength $400 \mathrm{mT} / \mathrm{m}$ ). For the preparation of ex vivo samples, we used our previous protocol, which guaranteed a good tissue quality for MRI analyses (Murgoci et al. 2019). A quadrature volume RF transceiver was used with diameter of $25 \mathrm{~mm}$ (STARK contrast, Erlangen, Germany). On the day of the ex vivo measurement the samples were removed out of the fixating solution and covered by plastic foil. Each MR session consisted of four samples placed together into RF coil to optimize the filling factor of the RF coil and to reduce overall acquisition time. The centres of the lesions were properly aligned.

First a fast spin echo sequence $(\mathrm{TR} / \mathrm{TE} e f f=2500 \mathrm{~ms} / 12 \mathrm{~ms}$; echo train length $=4$; echo spacing $=12 \mathrm{~ms} ; 1$ average; field of view $=25.4 \times 25.4 \mathrm{~mm}$; matrix size $=256 \times 256$; 16 slices; slice thickness $=2 \mathrm{~mm}$ without gap) was acquired to verify the position of the lesion within the isocenter of the RF coil.

A diffusion weighted (DW) fast spin echo sequence was then used to acquire one b0 image and six DW images with the b-value of 0 and $1500 \mathrm{~s} / \mathrm{mm}^{2}$, respectively. Six noncollinear diffusion directions were used $([0,0,0],[1 / \sqrt{ } 2$, $1 / \sqrt{ } 2,0],[0,1 / \sqrt{ } 2,1 / \sqrt{ } 2],[1 / \sqrt{ } 2,0,1 / \sqrt{ } 2],[1 / \sqrt{ } 2,-1 / \sqrt{ } 2,0],[0$, $-1 / \sqrt{ } 2,1 / \sqrt{ } 2],[-1 / \sqrt{ } 2,0,1 / \sqrt{ } 2])$ and the following acquisition parameters: TR/TEeff $=2500 \mathrm{~ms} / 32 \mathrm{~ms}$; echo train length $=4 ; \delta=4 \mathrm{~ms}, \Delta=12 \mathrm{~ms}$, echo spacing $=13.3 \mathrm{~ms} ; 28$ averages; field of view $25.6 \times 25.6 \mathrm{~mm}$ and matrix size of $256 \times 256$. A total of 16 axial slices of $2 \mathrm{~mm}$ thickness without gap were used to cover the $3 \mathrm{~cm}$ spine segment. The total acquisition time was $8 \mathrm{~h}$ and $43 \mathrm{~min}$. During the MR acquisition the temperature in the magnet bore was maintained at $22^{\circ} \mathrm{C}$ by warm air (SA Instruments, Inc.)

\section{DTI analysis}

Prior the analysis the raw data was zero-filled to $512 \times 512$ data matrix and smoothed in time domain with Gaussian filter in both the read and phase direction directly on MR console using VnmrJ 4.2 software. The image data were further transferred to DSI Studio (http://dsi-studio.labsolver. org) for subsequent diffusion tensor analysis.

\section{ROI selection}

Two regions of interest (ROIs) were selected on each slice. The first ROI was placed in the area of dorsal column. The second ROI encompasses the entire spinal cord. The selection of dorsal column was done manually on b0 images. The selection of entire spinal cord was done semi automatically on RD map giving the best contrast between the tissue and surrounding protein matrix using intensity thresholding and manual editing. The average values of DTI metrics (MD, FA, AD and RD) were evaluated from 
the selected ROIs at the level of a slice and whole spine segment. However, the data of ROIs encompassing dorsal column were not shown further due to little additional information. These data were only discussed. Since the exact position of the lesion epicentre was difficult to set before the MR experiment with sufficient accuracy the final position of the lesion epicentre was determined as slice with the lowest value of FA. This adjustment was performed on each spinal cord sample before further statistical analysis was applied.

\section{Statistical evaluation}

One-way analysis of variance (ANOVA) was used to evaluate the differences in DTI parametric measures between alginate/saline-treated and sham-operated group. Tukey's test for multiple comparisons was used for post hoc analysis with a level of significance $p<0.05$. Shapiro-Wilk test was used to test normality of the sample group. Levene's test was used to test the equality of variance between the groups. All tests were performed using free online statistical tools available on https://www.statskingdom.com/.

\section{Results}

Representative images of axial section of sham operated and injured rat spinal cord surrounded by protein matrix are shown in Figure 1 and 2. Signal to noise ratio (SNR) for white matter in sham rats was $33 \pm 3$ and $29 \pm 6$ for b0 image (diffusion weighting factor $\mathrm{b}=0 \mathrm{~s} / \mathrm{mm}^{2}$ ) and DW image (dif- fusion weighting factor $b=1500 \mathrm{~s} / \mathrm{mm}^{2}$ ), respectively. SNR calculation was performed on magnitude images directly on console without any post processing applied. The ROI was placed in the area of white matter and in the air area on each slice. The mean signal intensity values were calculated. The mean signal value for DW image was derived from all 6 DW images.

Clear separation of white and grey matter is apparent on b0 images as well as on derived parametric maps (Fig. 2). SCI produced characteristic changes in DTI measures with decreasing intensity from epicentre towards the distal ends. Decrease in FA and increase in MD are main hallmarks of disrupted tissue integrity seen around epicentre on respective derived maps (Fig. 2).

Distinction between sham operated group and injured group was evident on tractography images as well (Fig. 3). More tracts in sham operated group were clearly detected using a deterministic fiber tracking algorithm (Yeh et al. 2013). However, only visual inspection was performed on the tractography data without further track analysis. Therefore this parameter needs to be analysed in more detail in our future studies.

The significant difference between each of the treated groups relative to sham operated group was present for FA, MD and RD (ANOVA; $p<0.001$ ) for both ROIs at the level of entire spine. Similarly, the significant difference was present for all four DTI metrics at the slice level throughout the length of the spinal cord. The statistical significance compared to sham operated group was however not further stated and in the results we focused only on the differences between the saline $v s$. alginate-treated groups.

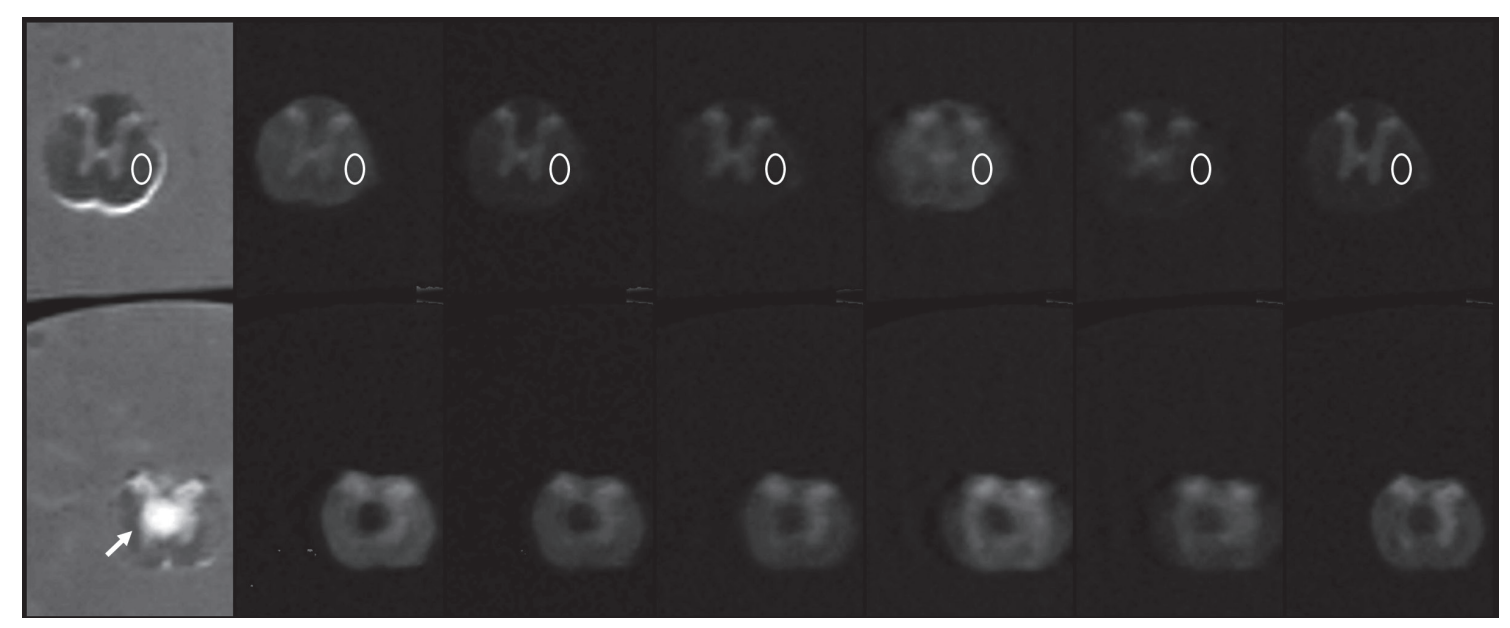

Figure 1. Axial slice of sham operated spinal cord (upper row) and spinal cord after contusion injury (lower row). First column is b0 image $\left(b=0 \mathrm{~s} / \mathrm{mm}^{2}\right)$ followed by six diffusion weighted images measured with $b$-value of $1500 \mathrm{~s} / \mathrm{mm}^{2}$. Data are shown without any post processing and with uniform intensity scaling. White ellipse shows the region of interest (ROI) used for signal to noise calculation from the white matter. The ROI from noise is not shown. The cyst filled with cerebrospinal fluid formed after necrosis is clearly visible in the central part of the injured spinal cord (white arrow). 


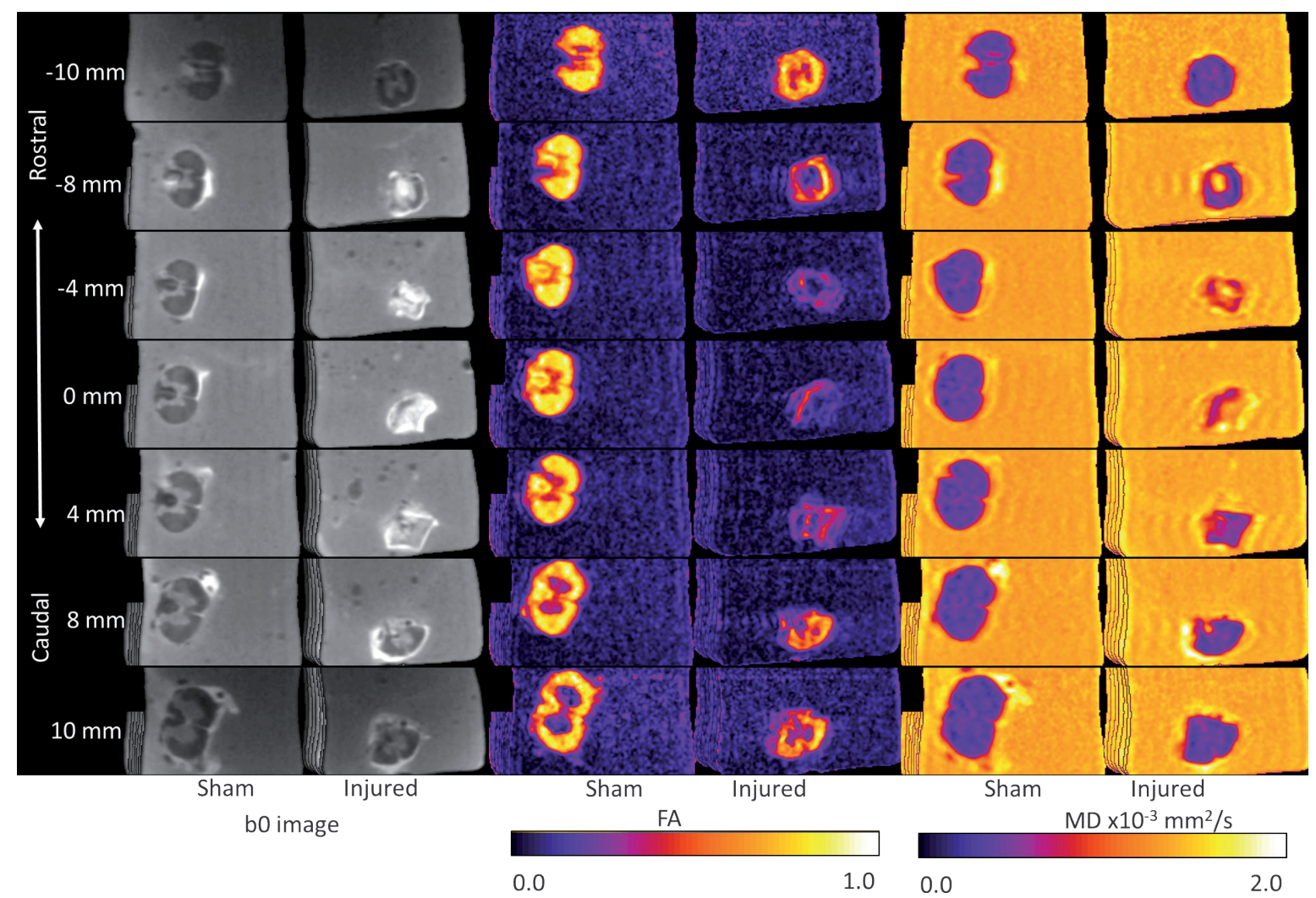

Figure 2. Diffusion tensor images of representative sham and injured spinal cord around lesion epicentre. b0 image (left), fractional anisotropy (FA) map (middle) and mean diffusivity (MD) map (right). Severe contusion injury resulted in structural changes spreading more than $10 \mathrm{~mm}$ in both directions from the lesion epicentre.

At the level of the spinal cord, we found significant increase in FA (ANOVA; $p<0.005)$ and decrease in RD (ANOVA; $p<0.05$ ) between alginate- and saline-treated group, respectively (Fig. 4). Similar changes were found at the level of entire dorsal column, however without statistical significance (the data are not shown). To analyse the changes in DTI measures along the length of spinal cord segment, the ROIs were analysed separately on each individual slice. Significant changes were found for individual ROIs encompassing entire spine area for all four parametric measures; however, the most frequent changes were detected in FA and RD (Fig. 5). Table 1 summarises individual statistical significances on selected slices.

Parametric measures from ROIs encompassing dorsal column did not reach statistical significances (the data not shown).

Interestingly, we observed a clear asymmetry in changes in DTI measures between the directions towards the rostral and caudal end from the epicentre (Fig. 5). After alginate treatment the rostral area seems to recover better compared to caudal direction. This difference was again more prominent on the data from entire spinal area.

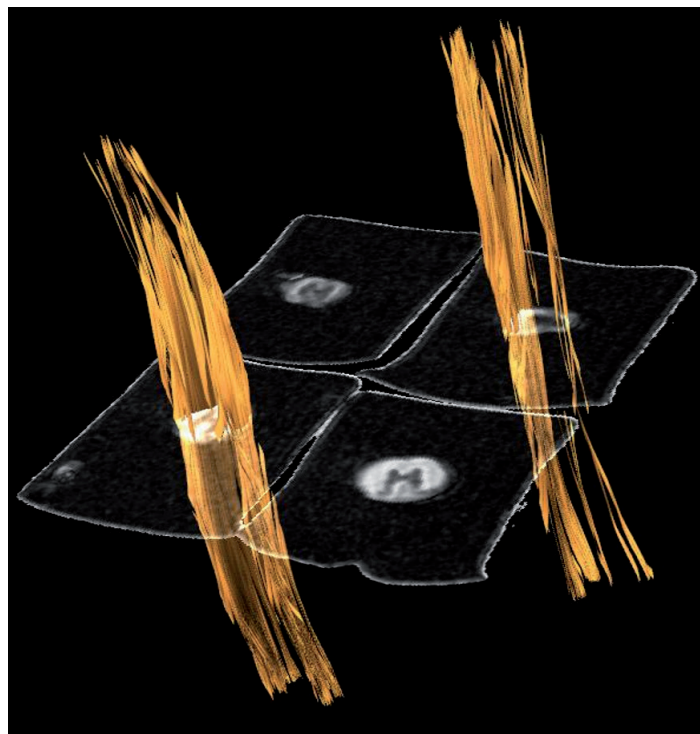

Figure 3. 3D visualization of white matter fiber tracts in the control spinal cord (left) and in the spinal cord after contusion injury (right). Fewer tracts were found in white matter of injured spinal cord using a deterministic fiber tracking algorithm. 


\section{Discussion}

In present study, we followed up on our previous experiments involving key growth factors released from the injected alginate biomaterial which significantly enhanced the sparing of spinal cord tissue and enhanced outgrowth of corticospinal tract axons, probably by stimulating endogenous neural stem cells. Thus, the results from current study suggest that DTI is able to detect the structural changes, which resulted from the alginate treatment in a severe contusion injury rat model. The main findings are increased FA and decreased RD in alginatetreated group compared to saline-treated group. These finding are consistent with presumed structural changes following successful treatment such as restoring axonal integrity and remyelination. Similar findings of increased FA after treatment were recently reported by Gao et al. (2020). Interestingly, based on the provided SNR data by Ellingson et al. (2008a), we managed to obtain comparable image quality using ex vivo DTI in magnetic field far below preclinical 9.4 T standard as was proposed by de Graff et al. (2006).
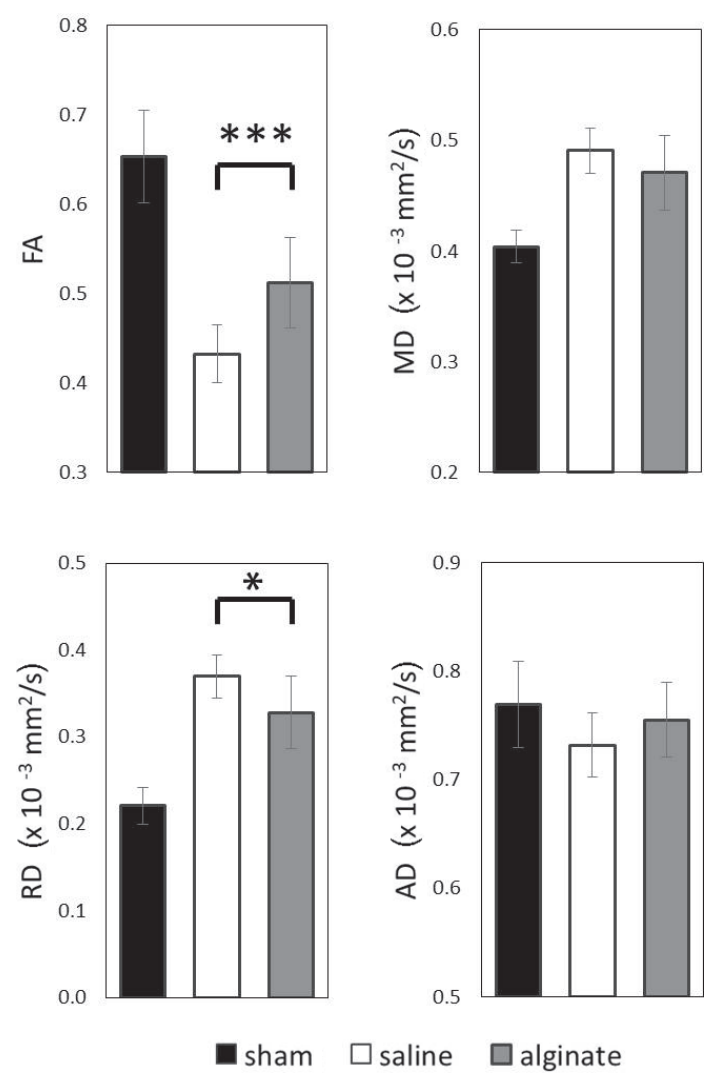

Figure 4. Averaged values of DTI parametric measures over the entire spinal cord segment. FA and RD were the most sensitive parameters of alginate treatment (ANOVA; ${ }^{*} p<0.05,{ }^{* * *} p<0.005$ ). Error bars indicate the standard deviation for each group. FA, fractional anisotropy; $\mathrm{MD}$, mean diffusivity; $\mathrm{RD}$, radial diffusivity; $\mathrm{AD}$, axial diffusivity.

\section{DTI metrics at the spine level}

DTI metrics at the spine level showed significant difference between the treated groups. However, our assumption that DTI metrics derived from the dorsal column should be susceptible to the tissue pathology has not been confirmed. The data from DC area were less statistically significant and subjected to substantial bias due to uncertainty in ROI selection and increased variance in ROI signal intensity. This bias was intensified especially in the severe contusion injury, where the lesion spreads almost across entire measured area (Fig. 2). These conclusions were actually already proposed in recent studies (Jirjis et al. 2013; Talbott et al. 2016) that the global ROIs are more reliable and give more statistical power comparing the white matter or grey matter ROI only. Moreover, it was suggested that the DTI metrics should not be confined to lesion area only. In fact, many studies showed DTI changes after SCI in distant part from the lesion even in the brain tissue (Jirjis et al. 2016).

Further, we found similar pattern in changes of all DTI metrics for both entire spinal cord and dorsal column. This

Table 1. Analysis of differences in DTI parameters at the slice level among groups

\begin{tabular}{|c|c|c|c|c|}
\hline \multirow{2}{*}{\multicolumn{2}{|c|}{ Position }} & \multirow{2}{*}{$\begin{array}{l}\text { DTI } \\
\text { parameter }\end{array}$} & \multicolumn{2}{|c|}{$p$ value } \\
\hline & & & $\begin{array}{c}\text { Sham } v s . \\
\text { Saline }\end{array}$ & $\begin{array}{c}\text { Saline } v s . \\
\text { Alginate }\end{array}$ \\
\hline \multirow{16}{*}{ 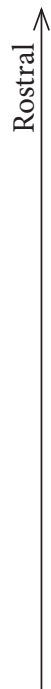 } & \multirow[t]{4}{*}{$-8 \mathrm{~mm}$} & FA & $<0.0001$ & $<0.05$ \\
\hline & & MD & $<0.005$ & n.s. \\
\hline & & $\mathrm{AD}$ & n.s. & n.s. \\
\hline & & $\mathrm{RD}$ & $<0.0005$ & $<0.05$ \\
\hline & \multirow[t]{4}{*}{$-4 \mathrm{~mm}$} & FA & $<0.0001$ & $<0.01$ \\
\hline & & MD & $<0.0001$ & $<0.01$ \\
\hline & & $\mathrm{AD}$ & $<0.05$ & $<0.05$ \\
\hline & & $\mathrm{RD}$ & $<0.0001$ & $<0.01$ \\
\hline & \multirow[t]{4}{*}{$0 \mathrm{~mm}$} & FA & $<0.0001$ & $<0.0005$ \\
\hline & & MD & $<0.0001$ & n.s. \\
\hline & & $\mathrm{AD}$ & $<0.05$ & n.s. \\
\hline & & $\mathrm{RD}$ & $<0.0001$ & $<0.05$ \\
\hline & \multirow[t]{4}{*}{$4 \mathrm{~mm}$} & FA & $<0.0001$ & $<0.05$ \\
\hline & & MD & $<0.001$ & n.s. \\
\hline & & $\mathrm{AD}$ & n.s. & n.s. \\
\hline & & $\mathrm{RD}$ & $<0.0001$ & n.s. \\
\hline \multirow{4}{*}{ 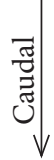 } & \multirow[t]{4}{*}{$8 \mathrm{~mm}$} & FA & n.s. & n.s. \\
\hline & & MD & n.s. & n.s. \\
\hline & & $\mathrm{AD}$ & n.s. & n.s. \\
\hline & & $\mathrm{RD}$ & n.s. & n.s. \\
\hline
\end{tabular}

Sham, sham-operated group; Saline, saline-treated group; Alginate, alginate-treated group. ANOVA with Tuckey post hoc analysis. DTI, diffusion tensor imaging; FA, fractional anisotropy; MD, mean diffusivity; $\mathrm{AD}$, axial diffusivity; $\mathrm{RD}$, radial diffusivity; n.s., test not significant. 

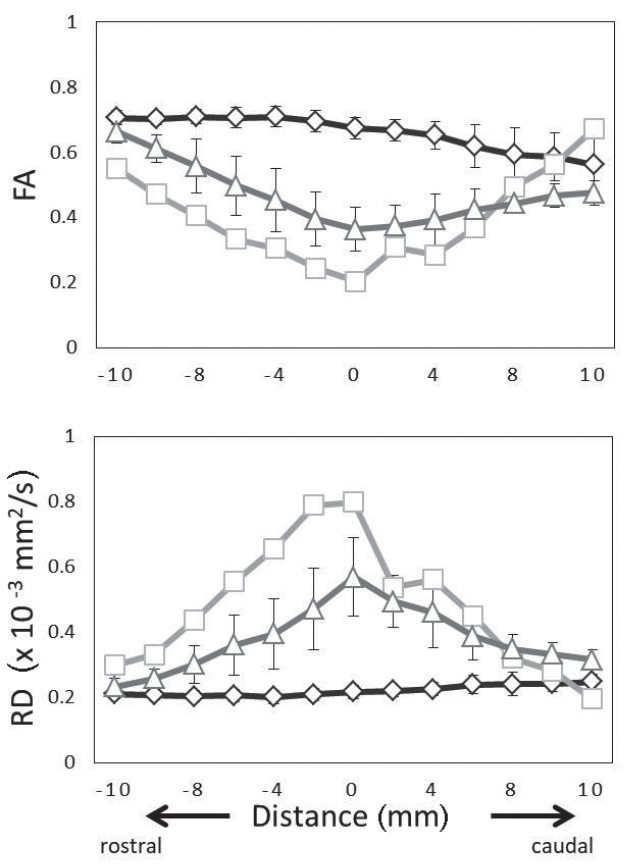

$\leadsto$ sham $-\square$-saline
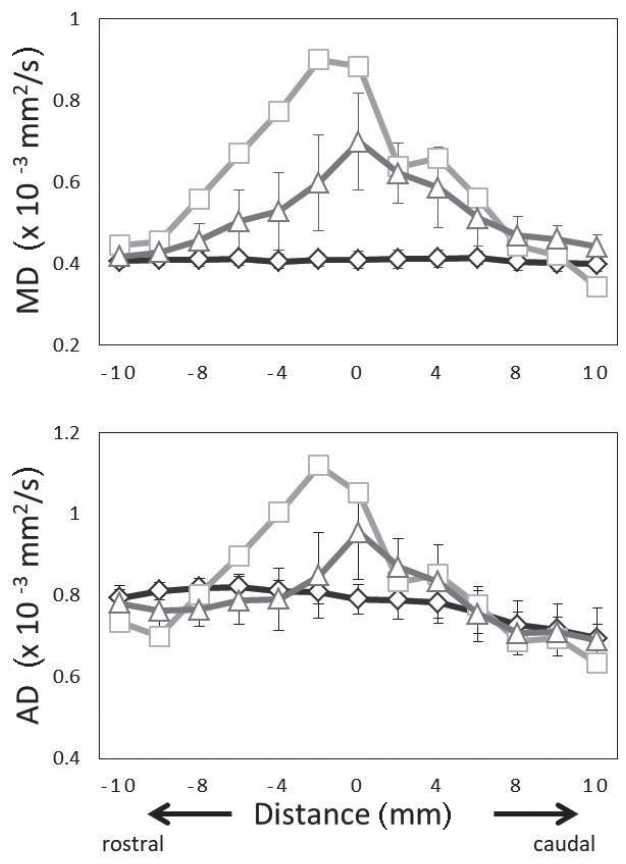

Figure 5. A comparison of DTI measures between alginate treated and saline treated group at the level of the slice. The data are taken from the ROI that includes both white and grey matter. The changes in diffusion parameters after alginate treatment are more prominent in the direction towards the rostral end of the spinal cord. Error bars not shown for salinetreated group to preserve graph readability. For abbreviations, see Fig. 4. is in accordance to similar diffusivity changes found in white and grey matter after SCI (Jirjis et al. 2013).

\section{DTI metrics at the slice level}

The contusion injury produced in the spinal cord characteristic changes in DTI measures over the length of the spinal cord. Decrease in FA and increase in RD, MD and $\mathrm{AD}$ were consistent with the previously reported findings after SCI (Ellingson et al. 2008a; Jirjis et al. 2013; Talbott et al. 2016). Parameters AD, RD and MD constantly decrease towards distal ends from epicentre. This drop is however not so prominent to reach the values published in previous studies (Ellingson et al. 2008a; Jirjis et al. 2013). The reason for this could be collecting the data from severe lesion over relative short distance from the epicentre limited by the RF coil. Interestingly, similar pattern was reported in our previous in vivo study of spine tissue recovery after moderate SCI (Murgoci et al. 2019).

Alginate treatment caused changes in $\mathrm{FA}, \mathrm{AD}, \mathrm{RD}$ and MD towards the sham DTI values (Fig. 5). However, statistical tests were conclusive only for FA and RD in whole cord ROI (Table 1). The data from the dorsal column did not reach statistical significance in any of the selected slices. This is most probably due to increased variability in signal intensity caused by the smaller size of the individual ROIs.

The observed asymmetry in DTI measures between rostral and caudal direction around lesion epicentre (Fig. 5) might be related to SCI procedure itself resulting in different extents of damage/sparing between the segments. However, similar asymmetric pattern was observed after SCI with varying degrees of intensity using external impactor (Jirjis et al. 2013). Furthermore, enhanced capacity of spontaneous regeneration in the rostral segments (above the lesion), where corticospinal and rubrospinal tracts remain connected with the brain stimulatory pathways may play a role as well (Grulova et al. 2015).

\section{Tractography}

Tractography based characteristics could be a promising approach for treatment efficacy assessment as well. In our study, however; only visual inspection of tract density was performed shoving clearly visible difference between injured and sham operated animal (Fig. 3). The same approach was used by Gao et al. (2020) to present only a visual qualitative parameter of applied treatment. However, tractography can provide additional set of parameters with respect to fiber length and fiber number together with tract-based ROI selection with following DTI metrics evaluation. This analysis was used to characterise the tissue recovery after SCI (Ellingson et al. 2008a).

Finally, the primary and secondary damage resulting from SCI is a complex event located throughout entire spinal cord. Even though DTI provides a close link towards tissue structure, one has to be cautious when interpreting 
DTI results without supporting histological or immunohistochemical findings.

\section{Conclusion}

Results from the current study demonstrated the feasibility of ex vivo DTI to be used to assess the efficacy of treatment after SCI. Especially, parameters FA and RD averaged over large spine regions showed as a robust biomarker of tissue recovery after SCI.

Acknowledgments. This was supported by APVV 19-0193, APVV19-0032, APVV 18-0515, VEGA 1/0376/20, VEGA 2/0003/20, VEGA-02/0166/20. The authors want to thank to Zuzana Rostakova, $\mathrm{PhD}$ (Institute of Measurement science, SAS) for her consultation during statistical analyses.

\section{References}

Cizkova D, Slovinska L, Grulova I, Salzet M, Cikos S, Kryukov O, Cohen S (2015): The influence of sustained dual-factor presentation on the expansion and differentiation of neural progenitors in affinity-binding alginate scaffolds. J. Tissue Eng. Regen. Med. 9, 918-929 https://doi.org/10.1002/term.1797

Cizkova D, Murgoci AN, Cubinkova V, Humenik F, Mojzisova Z, Maloveska M, Cizek M, Fournier I, Salzet M (2020): Spinal cord injury: animal models, imaging tools and the treatment strategies. Neurochem. Res. 45, 134-143 https://doi.org/10.1007/s11064-019-02800-w

Ellingson BM, Kurpad SN, Schmit BD (2008a): Ex vivo diffusion tensor imaging and quantitative tractography of the rat spinal cord during long-term recovery from moderate spinal contusion. J. Magn. Reson. Imaging 28, 1068-1079 https://doi.org/10.1002/jmri.21578

Ellingson BM, Schmit BD, Ulmer JL, Kurpad SN (2008b): Diffusion Tensor Magnetic Resonance Imaging In Spinal Cord Injury. Concepts In Magnetic Resonance Part A. Bridging Education and Research https://doi.org/10.1002/cmr.a.20112

Farrell JAD, Zhang J, Jones MV, DeBoy CA, Hoffman PN, Landman BA, Smith SA, Reich DS, Calabresi PA, Van Zijl PCM (2010): Q-space and conventional diffusion imaging of axon and myelin damage in the rat spinal cord after axotomy. Magn. Reson. Med. 63, 1323-1335 https://doi.org/10.1002/mrm.22389

Gao Z, Zhao Y, He X, Leng Z, Zhou X, Song H, Wang R, Gao Z, Wang Y, Liu J, et al. (2020): Transplantation of Sh-MiR-199a-5pmodified olfactory ensheathing cells promotes the functional recovery in rats with contusive spinal cord injury. Cell Transplant. 29, 0963689720916173 https://doi.org/10.1177/0963689720916173
Graaf R, Brown PB, McIntyre S, Nixon TW, Behar KL, Rothman DL (2006): High magnetic field water and metabolite proton $\mathrm{T} 1$ and T2 relaxation in rat brain in vivo. Magn. Reson. Med. 56, 386-394 https://doi.org/10.1002/mrm.20946

Grulova I, Slovinska L, Blaško J, Devaux S, Wisztorski M, Salzet M, Fournier I, Kryukov O, Cohen S, Cizkova D (2015): Delivery of alginate scaffold releasing two trophic factors for spinal cord injury repair. Sci. Rep. 5, 13702 https://doi.org/10.1038/srep13702

Jirjis MB, Kurpad SN, Schmit BD (2013): Ex vivo diffusion tensor imaging of spinal cord injury in rats of varying degrees of severity. J. Neurotrauma 30, 1577-1586 https://doi.org/10.1089/neu.2013.2897

Jirjis MB, Vedantam A, Budde MD, Kalinosky B, Kurpad SN (2016): Severity of spinal cord injury influences diffusion tensor imaging of the brain. J. Magn. Reson. Imaging 43, 63-74 https://doi.org/10.1002/jmri.24964

Jirjis MB, Valdez C, Vedantam A, Schmit BD, Kurpad SN (2017): Diffusion tensor imaging as a biomarker for assessing neuronal stem cell treatments affecting areas distal to the site of spinal cord injury. J. Neurosurg. Spine 26, 243-251 https://doi.org/10.3171/2016.5.SPINE151319

Kaushal M, Shabani S, Budde M, Kurpad SN (2019): Diffusion tensor imaging in acute spinal cord injury: a review of animal and human studies. J. Neurotrauma 36, 2279-2286 https://doi.org/10.1089/neu.2019.6379

Murgoci AN, Baciak L, Cubinkova V, Smolek T, Tvrdik T, Juranek I, Kafka J, Cizkova D (2019): Diffusion tensor imaging: tool for tracking injured spinal cord fibres in rat. Neurochem. Res. 45, 180-187 https://doi.org/10.1007/s11064-019-02801-9

Smith SA, Pekar JJ, Van Zijl PCM (2012): Advanced MRI strategies for assessing spinal cord injury. In: Handb. Clin. Neurol. 109, 85-101 https://doi.org/10.1016/B978-0-444-52137-8.00006-1

Talbott JF, Nout-Lomas YS, Wendland MF, Mukherjee P, Russell Huie J, Hess CP, Mabray MC, Bresnahan JC, Beattie MS (2016): Diffusion-weighted magnetic resonance imaging characterization of white matter injury produced by axon-sparing demyelination and severe contusion spinal cord injury in rats. J. Neurotrauma 33, 929-942 https://doi.org/10.1089/neu.2015.4102

Vanický I, Urdzíková L, Saganová K, Cízková D, Gálik J (2001): A simple and reproducible model of spinal cord injury induced by epidural balloon inflation in the rat. J. Neurotrauma 18, 1399-1407 https://doi.org/10.1089/08977150152725687

Yeh Fang C, Verstynen TD, Wang Y, Fernández-Miranda JC, Tseng WYI (2013): Deterministic diffusion fiber tracking improved by quantitative anisotropy. PLoS One 8, 1-16 https://doi.org/10.1371/journal.pone.0080713

Received: May 28. 2021

Final version accepted: August 13, 2021 